\title{
TESTING THE STABILITY OF REGRESSION PARAMETERS WHEN SOME ADDITIONAL DATA SETS ARE AVAILABLE
}

\author{
R. RADHAKRISHNAN \\ DON R. ROBINSON \\ Department of Management and Quantitative Methods \\ Illinois State University \\ Normal, Illinois 61761 \\ (Received June 2, 1992 and in revised form September 8, 1992)

\begin{abstract}
We consider the problem of testing the stability of regression parameters in regression lines of different populations when some additional, but unidentified, data sets from those populations are available. The standard test $\left(\mathrm{T}_{0}\right)$ discards the additional data and tests the stability of the regression parameters using only the data sets from identified populations. We propose two test procedures $\left(T_{1}\right.$ and $\left.T_{2}\right)$ utilizing all the available data, because the additional data may contain information about the parameters of the regression lines which are tested for stability. A power comparison among the tests is also presented. It is shown that $T_{1}$ always has larger power than $T_{0}$. In certain situations $T_{2}$ has the largest power.
\end{abstract}

KEY WORDS AND PHRASES: least squares estimates, regression parameters, power of the test.

1992 AMS SUBJECT CLASSIFICATION CODES: 62J05, 62J99

1. INTRODUCTION. Consider the regression model

$$
\mathrm{y}_{\mathrm{ij}}=\alpha_{\mathrm{i}}+\beta_{\mathrm{i}}\left(\mathrm{x}_{\mathrm{ij}}-\overline{\mathrm{x}}_{\mathrm{i}}\right)+\epsilon_{\mathrm{ij}}, \mathrm{i}=1,2, \ldots, \mathrm{k}, \mathrm{j}=1,2, \ldots, \mathrm{n}_{\mathrm{i}},
$$

where the $y_{\mathrm{ij}}$ are observations on the response variable, the $\mathrm{x}_{\mathrm{ij}}$ are observations on the predictor variable, $\alpha_{i}$ and $\beta_{i}$ are the regression parameters, and the $\epsilon_{\mathrm{ij}}$ are the error terms, which are unobserved random variables. It is assumed that the errors are independent, normally distributed random variables with mean 0 and common unknown variance $\sigma^{2}$. For the model, $\alpha_{i}+\beta_{i}\left(x_{i j}-\bar{x}_{i}\right)$ is the regression line of the variable $y$ on the predictor variable $x$ for the $i^{\text {th }}$ group, $\alpha_{i}$ is the $y$ intercept when $x=\bar{x}_{i}$, and $\beta_{i}$ is the slope. Suppose we have $m(m \leq k)$ additional data sets corresponding to $\mathrm{m}$ regression lines whose model is given by

$$
\mathrm{y}_{\mathrm{ij}}=\alpha_{\mathrm{i}}+\beta_{\mathrm{i}}\left(\mathrm{x}_{\mathrm{ij}}-\overline{\mathrm{x}}_{\mathrm{i}}\right)+\epsilon_{\mathrm{ij}}, \mathrm{i}=\mathrm{k}+1, \ldots, \mathrm{k}+\mathrm{m}, \mathrm{j}=1,2, \ldots, \mathrm{n}_{\mathrm{i}} \text {. }
$$

We assume that the error terms $\epsilon_{\mathrm{ij}}$ in model (1.2) are independent, normally distributed random variables with mean 0 and common unknown variance $\sigma^{2}$. It is further assumed that the $\mathrm{m}$ regression lines in model (1.2) are an unknown subset of the $\mathbf{k}$ regression lines in model (1.1). 
However, we cannot identify the $m$ regression lines associated with the additional data sets $\left(y_{i j}\right.$, $\left.\mathrm{x}_{\mathrm{ij}}, \mathrm{i}=\mathrm{k}+1, \ldots, \mathrm{k}+\mathrm{m}, \mathrm{j}=1,2, \ldots, \mathrm{n}_{\mathrm{i}}\right)$. We are interested in testing the null hypothesis $\mathrm{H}_{0}: \alpha_{1}=\alpha_{2}=\ldots=$ $\alpha_{\mathrm{k}} ; \beta_{1}=\beta_{2}=\ldots=\beta_{\mathrm{k}}$ against $\mathrm{H}_{\mathrm{a}}$ : either $\alpha_{\mathrm{i}} \neq \alpha_{\mathrm{i}}$, or $\beta_{\mathrm{i}} \neq \beta_{\mathrm{i}}$, for at least one pair (i,i'), where $\mathrm{i} \neq \mathrm{i}$, $\mathrm{i}, \mathrm{i}^{\prime}=1,2, \ldots, \mathrm{k}$, utilizing all the available data. The null hypothesis implies that all the $\mathrm{k}$ regression lines in model (1.1) are coincident whereas the alternative hypothesis is that at least two of the regression lines are different. The standard test $\left(T_{0}\right)$ of $H_{0}$ against $H_{a}$ using the $k$ data sets $\left(y_{i j}\right.$, $\left.x_{i j}, i=1,2, \ldots, k, j=1,2, \ldots, n_{i}\right)$ is well-known in the literature and has diverse applications. A biostatistician may be interested in testing the equivalence of regression lines for predicting the systolic blood pressure using age as the predictor variable for four social groups. A test for the stability of the regression parameters that generated the data sets is $\mathrm{H}_{0}: \alpha_{1}=\alpha_{2}=\alpha_{3}=\alpha_{4} ; \beta_{1}=$ $\beta_{2}=\beta_{3}=\beta_{4}$. If $\mathrm{H}_{0}$ is true, we use a single regression line based upon the four data sets for predicting systolic blood pressure using age as the predictor variable, Klienbaum and Kupper [1]. An economist might be interested in testing the equivalence of multiple regression models for predicting the gross domestic product using labor and capital as predictor variables for different time periods, Maddala [2].

In this paper we consider two tests $\left(\mathrm{T}_{1}\right.$ and $\left.\mathrm{T}_{2}\right)$ utilizing all the available data and make a power comparison between these two tests and the standard test which is based solely on the $k$ data sets relating to the regression lines whose parameters are tested for stability. In Section 2 we determine least squares estimates of the regression parameters to obtain the test statistics for the problem. The noncentrality parameter of the tests is derived in Section 3. In Section 4 we derive our proposed tests, $T_{1}$ and $T_{2}$. We illustrate and compare the power of all three tests in Section 5.

2. LEAST SQUARES ESTIMATES. Consider the sum

$$
\phi_{0}=\sum_{i=1}^{k+m} \underset{j=1}{n_{i}}\left(y_{i j}-\hat{\alpha}_{i}-\hat{\beta}_{i}\left(x_{i j}-\overline{x_{i}}\right)\right)^{2},
$$

where $\hat{\alpha}_{i}$ and $\hat{\beta}_{i}$ are the estimates of regression parameters $\alpha_{i}$ and $\beta_{i}(i=1,2, \ldots, k+m)$. The least squares estimates of the regression parameters are obtained by differentiating $\phi_{0}$ partially with respect to $\hat{\alpha}_{i}$ and $\hat{\beta}_{i}$ and then solving the resulting normal equations for $\hat{\alpha}_{i}$ and $\hat{\beta}_{i}$. It can be shown that the least squares estimates of $\alpha_{i}$ and $\beta_{i}$ are given by

$$
\hat{\alpha}_{i}=\sum_{j=1}^{n_{i}} y_{i j} / n_{i}
$$

and

$$
\hat{\beta}_{i}=\sum_{j=1}^{n_{i}} y_{i j}\left(x_{i j}-\overline{x_{i}}\right) / \sum_{j=1}^{n_{i}}\left(x_{i j}-\overline{x_{i}}\right)^{2} .
$$

Then $R_{0}^{\prime} 2$, the unconditional error sum of squares, is obtained by substituting $\hat{\alpha}_{i}$ and $\hat{\beta}_{i}$ given by (2.2) and (2.3) into $\phi_{0}$. It can be shown that

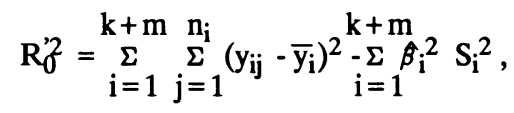

where 


$$
S_{i}^{2}=\sum_{j=1}^{n_{i}}\left(x_{i j}-\overline{x_{i}}\right)^{2}, i=1,2, \ldots, k+m
$$

The conditional error sum of squares under $\mathrm{H}_{0}$ is obtained by minimizing

$$
\phi_{1}=\sum_{i=1}^{k} \underset{j=1}{n_{i}}\left(y_{i j}-\hat{\alpha}-\hat{\beta}\left(x_{i j}-\bar{x}_{i}\right)\right)^{2} \underset{i=k+1}{+} \sum_{j=1}^{k+m}\left(y_{i j}-\hat{\alpha}_{i}-\hat{\beta}_{i}\left(x_{i j}-\bar{x}_{i}\right)\right)^{2}
$$

with respect to $\hat{\alpha}, \hat{\beta}, \hat{\alpha}_{\mathrm{i}}$, and $\hat{\beta}_{\mathrm{i}}(\mathrm{i}=\mathrm{k}+1, \ldots, \mathrm{k}+\mathrm{m})$.

The second sum on the right-hand side of (2.6) is minimized with respect to $\hat{\alpha}_{\mathrm{i}}$ and $\hat{\beta}_{\mathrm{i}}$ $(\mathrm{i}=\mathrm{k}+1, \ldots, \mathrm{k}+\mathrm{m})$ where $\hat{\alpha}_{\mathrm{i}}$ and $\hat{\beta}_{\mathrm{i}}$ are defined, respectively, as in equations (2.2) and (2.3). The least squares estimates of the regression parameters $\alpha$ and $\beta$ are given by

$$
\begin{gathered}
\hat{\alpha}=\underset{i=1}{\sum} \underset{j=1}{\sum_{i}} y_{i j} / n=\bar{y} \\
\hat{\beta}=\sum_{i=1}^{k} \underset{j=1}{n_{i}} y_{i j}\left(x_{i j}-\overline{x_{i}}\right) / \underset{i=1}{k} S_{i}^{2}
\end{gathered}
$$

where

$$
n=\underset{i=1}{k} n_{i}
$$

The conditional error sum of squares under $\mathrm{H}_{0}$ is

$$
R_{1}^{2}=\sum_{i=1}^{k} \underset{j=1}{n_{i}}\left(y_{i j}-\bar{y}\right)^{2}-\hat{\beta}^{2} \underset{i=1}{k} S_{i}^{2} \underset{i=k+1}{+\sum} \underset{j=1}{\sum}\left(y_{i j}-\overline{y_{i}}\right)^{2} \underset{i=k+m}{k+m} \hat{\beta}_{i}^{2} S_{i}^{2}
$$

The sum of squares for testing the null hypothesis $\mathrm{H}_{0}$ is

$$
\begin{aligned}
& \mathrm{SSH}_{0}=\mathrm{R}_{1}^{2}-\mathrm{R}_{\mathbf{0}}^{2} \\
& =\sum_{i=1}^{k} n_{i}\left(\overline{y_{i}}-\bar{y}\right)^{2}+\underset{i=1}{\sum} \hat{\beta}_{i}^{2} S_{i}^{2}-\beta^{2} \underset{i=1}{k} S_{i}^{2}, \\
& =\sum_{i=1}^{k} n_{i}\left(\overline{y_{i}}-\bar{y}\right)^{2}+\underset{i=1}{\sum} S_{i}^{2}\left(\hat{\beta}_{i}-\hat{\beta}\right)^{2},
\end{aligned}
$$

where

$$
\hat{\beta}=\sum_{i=1}^{k} S_{i}^{2} \hat{\beta}_{i} / \sum_{i=1}^{k} S_{i}^{2}
$$

It is well-known in the literature that $\mathrm{R}_{0}^{2} / \sigma^{2}$ is distributed as chi-square with

$$
\mathrm{n}^{\prime}=\mathrm{n}+\underset{\mathrm{i}=\mathrm{k}+1}{\mathrm{k}+\mathrm{m}} \mathrm{n}_{\mathrm{i}}-2(\mathrm{k}+\mathrm{m})
$$


degrees of freedom and $\mathrm{SSH}_{0} / \sigma^{2}$ is distributed as noncentral chi-square with $2(\mathrm{k}-1)$ degrees of freedom. When $\mathrm{H}_{0}$ is true, $\mathrm{SSH}_{0} / \sigma^{2}$ is distributed as chi-square with $2(\mathrm{k}-1)$ degrees of freedom. Further, $\mathrm{R}_{0}^{\prime 2}$ and $\mathrm{SSH}_{0}$ are independent; for example see Kshirsagar [3].

3. NONCENTRALITY PARAMETER. Here we derive the expected value of $\mathrm{SSH}_{0}$ under the non-null case. It can be shown that

$$
\sum_{i=1}^{k} n_{i}\left(\bar{y}_{i}-\bar{y}\right)^{2}=\sum_{i=1}^{k} n_{i}\left(\alpha_{i}-\bar{\alpha}\right)^{2}+\sum_{i=1}^{k} n_{i}\left(\bar{\epsilon}_{i}-\bar{\epsilon}\right)^{2}+2 \underset{i=1}{k} n_{i}\left(\alpha_{i}-\bar{\alpha}\right)\left(\overline{\epsilon_{i}}-\bar{\epsilon}\right),
$$

where

$$
\begin{aligned}
& \bar{\alpha}=\sum_{\mathrm{i}=1}^{\mathrm{k}} \mathrm{n}_{\mathrm{i}} \alpha_{\mathrm{i}} / \mathrm{n}, \\
& \overline{\epsilon_{\mathrm{i}}}=\sum_{\mathrm{i}=1}^{\mathrm{k}} \epsilon_{\mathrm{ij}} / \mathrm{n}_{\mathrm{i}}, \\
& \bar{\epsilon}=\sum_{\mathrm{i}=1}^{\mathrm{k}} \mathrm{n}_{\mathrm{i}} \overline{\epsilon_{\mathrm{i}}} / \mathrm{n} .
\end{aligned}
$$

Taking expectations of both sides of (3.1) we obtain

$$
\mathrm{E}\left(\sum_{\mathrm{i}=1}^{\mathrm{k}} \mathrm{n}_{\mathrm{i}}\left(\overline{\mathrm{y}}_{\mathrm{i}}-\overline{\mathrm{y}}\right)^{2}\right)=\sum_{\mathrm{i}=1}^{\mathrm{k}} \mathrm{n}_{\mathrm{i}}\left(\alpha_{\mathrm{i}}-\bar{\alpha}\right)^{2}+(\mathrm{k}-1) \sigma^{2} .
$$

Now

$$
\begin{aligned}
\mathrm{E}\left(\sum_{\mathrm{i}=1}^{\mathrm{k}} \hat{\beta}_{\mathrm{i}}^{2} \mathrm{~S}_{\mathrm{i}}^{2}\right) & =\underset{\mathrm{i}=1}{\mathrm{k}} \mathrm{S}_{\mathrm{i}}^{2}\left(\sigma^{2} / \mathrm{S}_{\mathrm{i}}^{2}+\beta_{\mathrm{i}}^{2}\right) \\
& =\mathrm{k} \boldsymbol{\sigma}^{2}+\underset{\mathrm{i}=1}{\mathrm{k} \beta_{\mathrm{i}}^{2} \mathrm{~S}_{\mathrm{i}}^{2},}
\end{aligned}
$$

and

$$
\begin{aligned}
\mathrm{E}\left(\hat{\beta}^{2} \underset{\mathrm{i}=1}{\mathrm{k}} \mathrm{S}_{\mathrm{i}}^{2}\right) & =\sum_{\mathrm{i}=1}^{\mathrm{k}} \mathrm{S}_{\mathrm{i}}^{2}\left(\sigma^{2} /{\left.\underset{\mathrm{i}=1}{\sum} \mathrm{S}_{\mathrm{i}}^{2}+\bar{\beta}^{2}\right)}^{\mathrm{k}}\right. \\
& =\sigma^{2}+\bar{\beta}^{2} \underset{\mathrm{i}=1}{\mathrm{k}} \mathrm{S}_{\mathrm{i}}{ }^{2},
\end{aligned}
$$

where

$$
\bar{\beta}=\sum_{i=1}^{k} S_{i}^{2} \beta_{i} / \sum_{i=1}^{k} S_{i}^{2}
$$

Using equations (3.5), (3.6), (3.7), and (2.11) we get

$$
\mathrm{E}\left(\mathrm{SSH}_{0}\right)=2(\mathrm{k}-1) \sigma^{2}+\sum_{\mathrm{i}=1}^{\mathrm{k}} \mathrm{n}_{\mathrm{i}}\left(\alpha_{\mathrm{i}}-\bar{\alpha}\right)^{2}+\underset{\mathrm{i}=1}{\mathrm{k}} \mathrm{S}_{\mathrm{i}}^{2}\left(\beta_{\mathrm{i}}-\bar{\beta}\right)^{2} .
$$


Since $\mathrm{SSH}_{0} / \sigma^{2}$ is distributed as noncentral chi-square with $2(\mathrm{k}-1)$ degrees of freedom, it follows from (3.9) that the noncentrality parameter is given by

$$
\lambda=\left(1 / \sigma^{2}\right)\left[\sum_{i=1}^{k} n_{i}\left(\alpha_{i}-\bar{\alpha}\right)^{2}+\sum_{i=1}^{k} S_{i}^{2}\left(\beta_{i}-\bar{\beta}\right)^{2}\right] .
$$

4. TEST PROCEDURES. The standard procedure for testing $\mathbf{H}_{0}$ against $\mathbf{H}_{\mathbf{a}}$ is an F-test based upon the test statistic

$$
F_{0}=\left(\mathrm{SSH}_{0} / 2(\mathrm{k}-1)\right) /\left(\mathrm{R}_{0}^{2} /(\mathrm{n}-2 \mathrm{k})\right)
$$

where

$$
R_{0}^{2}=\sum_{i=1}^{k} \underset{j=1}{n_{i}}\left(y_{i j}-\overline{y_{i}}\right)^{2}-\underset{i=1}{k} \hat{\beta}_{i}^{2} S_{i}^{2}
$$

See, for example, Kshirsagar [3]. The above test rejects the null hypothesis $\mathbf{H}_{0}$ if $\mathrm{F}_{0}>$ $F_{\alpha, 2(k-1), n-2 k}$ and accepts $H_{0}$ otherwise, where $F_{\alpha, f_{1}, f_{2}}$ is the upper $100 \alpha$ percentile point of the F-distribution with $f_{1}$ numerator degrees of freedom (ndf) and $f_{2}$ denominator degrees of freedom (ddf). We note that the standard test is based upon the $k$ data sets $\left(y_{i j}, x_{i j}, i=1,2, \ldots, k\right.$, $\left.\mathrm{j}=1,2, \ldots, \mathrm{n}_{\mathrm{i}}\right)$ and discards the additional data $\left(\mathrm{y}_{\mathrm{ij}}, \mathrm{x}_{\mathrm{ij}}, \mathrm{i}=\mathrm{k}+1, \ldots, \mathrm{k}+\mathrm{m}, \mathrm{j}=1,2, \ldots, \mathrm{n}_{\mathrm{i}}\right)$.

Consider the following test procedure $\left(T_{1}\right)$. Reject $H_{0}$ if

$$
F_{1}=\left(S_{0} / 2(k-1)\right) /\left(R_{0}^{2} / n^{\prime}\right)>F_{\alpha, 2(k-1), n^{\prime}}
$$

and accept $\mathrm{H}_{0}$ otherwise. A comparison between $\mathrm{T}_{0}$ and $\mathrm{T}_{1}$ shows that both have the same ndf but that the latter has larger ddf than $T_{0}$. We further note that $T_{1}$ is based upon all the available data. Under the non-null case, both test statistics have noncentral F-distributions with the same noncentrality parameter $\lambda$ as in (3.10). Therefore $F_{1}$ will have larger power than $F_{0}$, Graybill [4].

When the $\mathrm{m}$ regression lines in (1.2) are an unidentified subset of the $\mathrm{k}$ regression lines in the model (1.1), testing $\mathrm{H}_{0}$ against $\mathrm{H}_{a}$ is equivalent to testing $H_{0}^{\prime}$ : the $k+m$ regression lines are identical against $\mathrm{H}_{\mathrm{a}}^{\prime}$ : at least two of them are different.

Following the procedure outlined in Section 2, it can be shown that the sum of squares for testing $\mathbf{H}_{\mathbf{b}}$ is

$$
\operatorname{SSH}_{0}^{\prime}=\underset{i=1}{k+m} n_{i}\left(\hat{\alpha}_{i}-\hat{\alpha}^{\prime}\right)^{2}+\underset{i=1}{\sum} S_{i}\left(\hat{\beta}_{i}-\hat{\beta}^{\prime}\right)^{2},
$$

where

$$
\begin{gathered}
\hat{\alpha}^{\prime}=\sum_{i=1}^{k+m} n_{i} \bar{y}_{i} / \sum_{i=1}^{k+m} n_{i}, \\
\hat{\beta}^{\prime}=\sum_{i=1}^{k+m} S_{i}^{2} \hat{\beta}_{i} / \sum_{i=1}^{k+m} S_{i}^{2} .
\end{gathered}
$$

The sampling distribution of $\mathrm{SSH}_{\mathrm{j}}^{\prime} / \sigma^{2}$, when $\mathrm{H}_{\mathrm{a}}^{\prime}$ is true, is noncentral chi-square with $2(\mathrm{k}+\mathrm{m}-1)$ degrees of freedom and noncentrality parameter 


$$
\lambda^{\prime}=\left(1 / \sigma^{2}\right)\left[\sum_{i=1}^{k+m} n_{i}\left(\alpha_{i}-\alpha\right)^{2}+\sum_{i=1}^{k+m} S_{i}^{2}\left(\beta_{i}-\beta\right)^{2}\right],
$$

where

$$
\alpha=\sum_{i=1}^{k} n_{i} \alpha_{i} / \sum_{i=1}^{k} n_{i}
$$

and

$$
\beta=\sum_{\mathrm{i}=1}^{\mathrm{k}+\mathrm{m}} \mathrm{S}_{\mathrm{i}}^{2} \beta_{\mathrm{i}} / \sum_{\mathrm{i}=1}^{\mathrm{k}+\mathrm{m}} \mathrm{S}_{\mathrm{i}}^{2}
$$

We note that $\mathrm{SSH}_{0}^{\prime}$ can be obtained from $\mathrm{SSH}_{0}$ by replacing $\mathrm{k}$ with $\mathrm{k}+\mathrm{m}$. When $\mathrm{H}_{0}^{\prime}$ is true, the sampling distribution of $\mathrm{SSH}_{0} / \sigma^{2}$ is chi-square with $2(\mathrm{k}+\mathrm{m}-1)$ degrees of freedom. Further, $\mathrm{SSH}_{0}$ and $\mathrm{R}_{0}^{2}$ are independent.

We use an F-test $\left(\mathrm{T}_{2}\right)$ to test $\mathrm{H}_{0}^{\prime}$ against $\mathrm{H}_{\mathrm{a}}^{\prime}$ based upon the test statistic

$$
\mathrm{F}_{2}=\left(\mathrm{SSH}_{0}^{\prime} / \mathrm{f}_{1}^{\prime}\right) /\left(\mathrm{R}_{0}^{\prime 2} / \mathrm{n}^{\prime}\right) \text {, }
$$

where $f_{1}^{\prime}=2(k+m-1)$. We reject $H_{0}^{\prime}$ if $F_{2}>F_{\alpha, f_{1}^{\prime}, n^{\prime}}^{\prime}$ and accept $H_{0}^{\prime}$ otherwise. When $H_{a}^{\prime}$ is true, the sampling distribution of $F_{2}$ is noncentral $F$ with $f_{1}^{\prime}$ ndf and $n$ ' ddf and noncentrality parameter $\lambda$ '. We use noncentral F-distribution tables to compute the power of the tests. The next section illustrates and compares the power of these three tests.

5. POWER COMPARISONS OF THE TESTS. When $\mathrm{H}_{0}$ and $\mathrm{H}_{0}$ are not true the test statistics (4.1), (4.3), and (4.10) follow noncentral F-distributions. The non-null distributions of the test statistics $F_{0}$ and $F_{1}$ have the same noncentrality parameter, $\lambda$, defined in (3.10). The noncentrality parameter for the non-null distribution of $F_{2}$ is $\lambda^{\prime}$ as defined in (4.7). The ndf for both $T_{0}$ and $T_{1}$ is $f_{1}=2(k-1)$. For $T_{2}$ the ndf is $f_{1}=2(k+m-1)$. $T_{0}$ has ddf $f_{2}=n-2 k$, while the ddf for $T_{1}$ and $T_{2}$ is $n$ ' as defined in (2.13).

Tables 1, 2, and 3 illustrate the powers of $T_{0}$ and our proposed tests, $T_{1}$ and $T_{2}$. We chose $\alpha=0.05$ and situations involving $\mathrm{k}=4$ regression lines. The number of data sets considered from unidentified populations is $m$, where $1 \leq \mathrm{m} \leq \mathrm{k}$. For simplicity we use equal sample sizes $\left(n_{i}=10\right)$ for the $k$ identified populations and equal sample sizes $\left(n_{i}^{*}\right)$ for the $m$ unidentified populations. From our earlier notation $n_{i}^{*}=n_{k+i}(i=1, \ldots, m)$. Tables 1,2 , and 3 differ in the magnitude of $n_{i}^{*}$.

In the tables we denote the noncentrality parameter for the power of test $T_{i}$ as $\lambda_{i}$. The power of each test is a function of $\lambda_{i}$ and the relevant degrees of freedom. As indicated above, $\lambda_{0}$ $=\lambda_{1}$. For $m=k$, each $\lambda_{i}$ is a specific value. For $m<k, \lambda_{0}$ and $\lambda_{1}$ are (the same) specific values, but $\lambda_{2}$ varies depending upon which unidentified populations produce the $\mathrm{m}$ data sets. For this reason we calculate the tests' powers for selected sets of $k$ regression lines and values of $S_{i}{ }^{2}$. The differences between the parameters of these lines together with $\mathrm{S}_{\mathrm{i}}{ }^{2}$ and $\sigma^{2}$ affect $\lambda_{\mathrm{i}}$. The parameters of the $\mathrm{k}$ lines, $\mathrm{S}_{\mathrm{i}}{ }^{2}$, and $\sigma^{2}$ were chosen to produce the three values indicated for $\lambda_{0}$, so that the power of $T_{0}$ is about $.25, .5$, and .75. If $T_{0}$ has very small power, then additional data provide very little improvement. Conversely, when the power of $T_{0}$ is very large, there is little need for improvement with additional data.

Examinations of the tables produce the following observations. The powers of $T_{0}$ are the same in all three tables because this test ignores the additional data sets. For a given $\lambda_{0}\left(\right.$ and $\left.\lambda_{1}\right)$ 
the power of $T_{1}$ is always greater than the power of $T_{0}$ consistent with Graybill's conclusion [4] that for a given ndf the power of the test increases as the ddf increases. Also, in each table the power of $T_{1}$ increases as $m$ increases, because the ndf remains at $2(k-1)$ while the ddf increases by $n_{i}^{*}-2$. Likewise, for each value of $m$ the power of $T_{1}$ increases from Table 1 through Table 3 because the ddf increases as a result of the $n_{i}^{*}$ increasing from 5 to 7 and finally to 10 .

The power of $T_{2}$ is heavily influenced by the choice of regression lines for the additional data when $\mathrm{m}<\mathrm{k}$. In each table the power of $\mathrm{T}_{2}$ does not consistently increase as $\mathrm{m}$ increases. The increases in $\lambda_{2}$ and ddf are sometimes offset by the increase in $f_{1}^{\prime}$ of $2 \mathrm{~m}$. For each value of $m$ the power of $T_{2}$ generally increases from Table 1 through Table 3 because of the same increase in ddf as for $T_{1}$. But as Table 1 indicates, for small $n_{i}^{*}$ relative to $n_{i}$ the power of $T_{2}$ may be lower than the power of $T_{0}$, and is seldom much better than the power of $T_{1}$. In Table 2 when the $n_{i}^{*}$ approaches $n_{i}$ in size, improvements in the power of $T_{2}$ over the power of $T_{1}$ are noticeable. Table 3 indicates that when the $n_{i}^{*}$ equal $n_{i}, T_{2}$ is superior to the power of $T_{1}$ except occasionally for small $\mathrm{m}$.

6. APPLICATIONS. Using additional data from unidentified populations improves the power of the test for stability of the parameters in $\mathrm{k}$ regression lines. The only requirement is that the error terms of the regression lines from all populations have a common variance. The power of our proposed test, $T_{1}$, is always greater than the power of the standard test, $T_{0}$. If $m$, the number of data sets from unidentified populations, is close to $k$ and if the $n_{i}^{*}$ are near the $n_{i}$, then $T_{2}$ can produce a larger increase in the power than $T_{1}$. If $m$ is small or if $n_{i}^{*}$ is small relative to $n_{i}$, then $T_{1}$ may be a better choice than $T_{2}$.

\begin{tabular}{crcccc}
\hline & \multicolumn{3}{c}{ noncentrality parameters } & \multicolumn{3}{c}{ Power of the tests } \\
$\mathrm{m}$ & $\lambda_{0}, \lambda_{1}$ & $\lambda_{2}$ & $\mathrm{~T}_{0}$ & $\mathrm{~T}_{1}$ & $\mathrm{~T}_{2}$ \\
\hline 4 & 4.454 & 6.681 & .2502 & .2622 & .2409 \\
4 & 9.085 & 13.627 & .5016 & .5252 & .5069 \\
4 & 14.866 & 22.299 & .7500 & .7750 & .7744 \\
3 & 4.454 & 5.707 to 6.440 & .2502 & .2598 & .2233 to .2518 \\
3 & 9.085 & 12.012 to 12.770 & .5016 & .5205 & .4803 to .5105 \\
3 & 14.866 & 19.191 to 21.353 & .7500 & .7701 & .7299 to .7858 \\
2 & 4.454 & 4.895 to 6.240 & .2502 & .2570 & .2115 to .2684 \\
2 & 9.085 & 10.648 to 12.055 & .5016 & .5151 & .4645 to .5243 \\
2 & 14.866 & 16.601 to 20.564 & .7500 & .7645 & .6944 to .8050 \\
1 & 4.454 & 4.650 to 5.248 & .2502 & .2539 & .2256 to .2536 \\
1 & 9.085 & 9.784 to 10.405 & .5016 & .5089 & .4738 to .5029 \\
1 & 14.866 & 15.637 to 17.399 & .7500 & .7579 & .7139 to .7687 \\
\hline
\end{tabular}

Table 1

Power of the F-tests for $\alpha=.05 \quad k=4 \quad n_{i}=10 \quad n_{i}^{*}=5$ 


\begin{tabular}{lrcccc}
\hline & \multicolumn{3}{c}{ noncentrality parameters } & \multicolumn{3}{c}{ Power of the tests } \\
$\mathrm{m}$ & $\lambda_{0}, \lambda_{1}$ & \multicolumn{1}{c}{$\lambda_{2}$} & $\mathrm{~T}_{0}$ & $\mathrm{~T}_{1}$ & \multicolumn{1}{c}{$\mathrm{T}_{2}$} \\
\hline 4 & 4.454 & 7.572 & .2502 & .2674 & .2836 \\
4 & 9.085 & 15.444 & .5016 & .5353 & .5914 \\
4 & 14.866 & 25.273 & .7500 & .7851 & .8522 \\
3 & 4.454 & 6.178 to 7.228 & .2502 & .2643 & .2481 to .2913 \\
3 & 9.085 & 13.127 to 14.217 & .5016 & .5294 & .5388 to .5810 \\
3 & 14.866 & 20.826 to 23.919 & .7500 & .7792 & .7874 to .8529 \\
2 & 4.454 & 5.071 to 6.954 & .2502 & .2606 & .2228 to .3057 \\
2 & 9.085 & 11.287 to 13.243 & .5016 & .5221 & .5014 to .5832 \\
2 & 14.866 & 17.295 to 22.843 & .7500 & .7718 & .7271 to .8616 \\
1 & 4.454 & 4.717 to 5.519 & .2502 & .2560 & .2310 to .2692 \\
1 & 9.085 & 10.022 to 10.854 & .5016 & .5131 & .4900 to .5287 \\
1 & 14.866 & 15.900 to 18.261 & .7500 & .7624 & .7283 to .7977 \\
\hline
\end{tabular}

Table 2

Power of the F-tests for $\alpha=.05 \quad k=4 \quad n_{i}=10 \quad n_{i}^{*}=7$

\begin{tabular}{lrcccc}
\hline & \multicolumn{3}{c}{ noncentrality parameters } & \multicolumn{3}{c}{ Power of the tests } \\
$\mathrm{m}$ & $\lambda_{0}, \lambda_{1}$ & \multicolumn{1}{c}{$\lambda_{2}$} & $\mathrm{~T}_{0}$ & $\mathrm{~T}_{1}$ & $\mathrm{~T}_{2}$ \\
\hline 4 & 4.454 & 8.908 & .2502 & .2730 & .3498 \\
4 & 9.085 & 18.170 & .5016 & .5459 & .7024 \\
4 & 14.866 & 29.732 & .7500 & .7955 & .9270 \\
3 & 4.454 & 6.867 to 8.404 & .2502 & .2695 & .2851 to .3522 \\
3 & 9.085 & 14.776 to 16.373 & .5016 & .5393 & .6189 to .6756 \\
3 & 14.866 & 23.220 to 27.749 & .7500 & .7891 & .8539 to .9207 \\
2 & 4.454 & 5.336 to 8.026 & .2502 & .2650 & .2395 to .3632 \\
2 & 9.085 & 12.230 to 15.025 & .5016 & .5306 & .5540 to .6643 \\
2 & 14.866 & 18.336 to 26.262 & .7500 & .7805 & .7703 to .9210 \\
1 & 4.454 & 4.807 to 5.883 & .2502 & .2589 & .2383 to .2909 \\
1 & 9.085 & 10.343 to 11.461 & .5016 & .5188 & .5119 to .5633 \\
1 & 14.866 & 16.254 to 19.425 & .7500 & .7683 & .7470 to .8330 \\
\hline
\end{tabular}

Table 3

Power of the F-tests for $\alpha=.05 \quad k=4 \quad n_{i}=10 \quad n_{i}^{*}=10$

\section{REFERENCES}

[1] KLIENBAUM, D. G. and L. L. KUPPER, Applied Regression and Other Multivariable Methods, Chapter 13, PWS-Kent, Boston, MA, 1978.

[2] MADDALA, G. S., Introduction to Econometrics, Chapter 4, MacMillian, New York, NY, 1988.

[3] KSHIRSAGAR, A. M., A Course in Linear Models, Chapter 3, Marcel Dekker, New York, NY, 1983.

[4] GRAYBILL, F. A., Theory and Applications of Linear Models, Chapter 4, Duxbury Press, Boston, MA, 1976. 


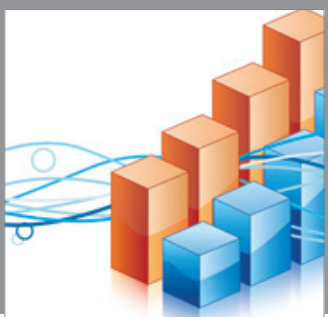

Advances in

Operations Research

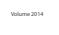

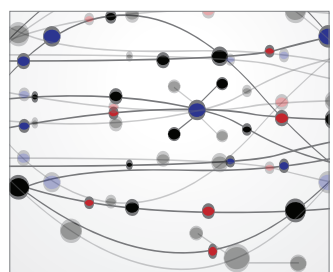

\section{The Scientific} World Journal
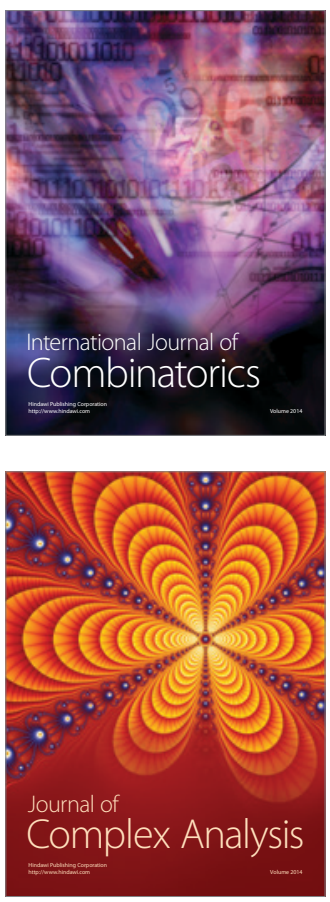

International Journal of

Mathematics and

Mathematical

Sciences
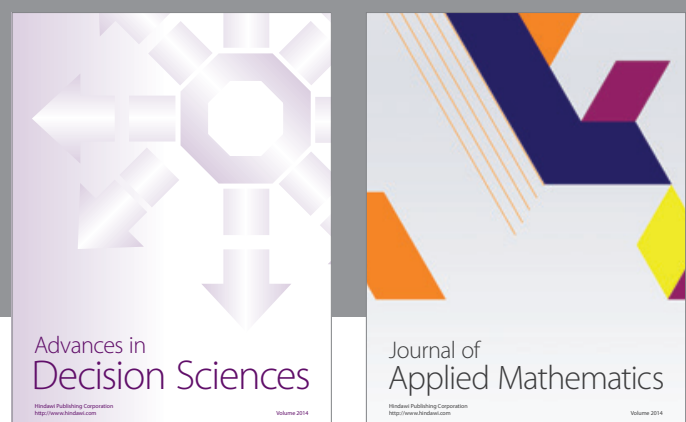

Journal of

Applied Mathematics
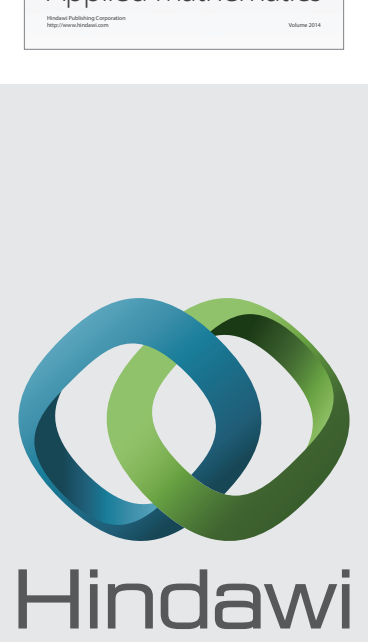

Submit your manuscripts at http://www.hindawi.com
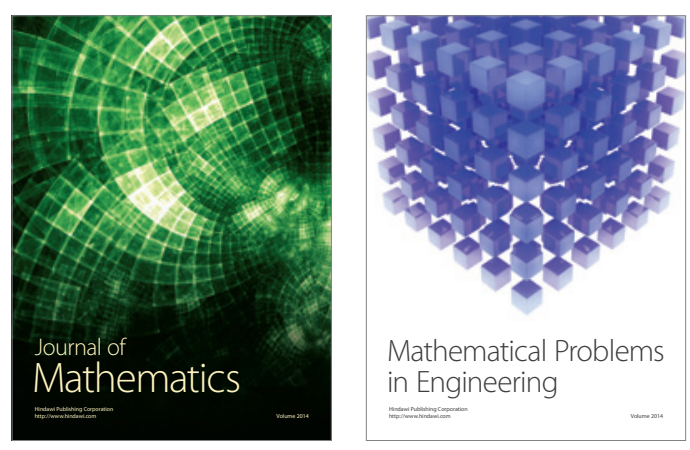

Mathematical Problems in Engineering
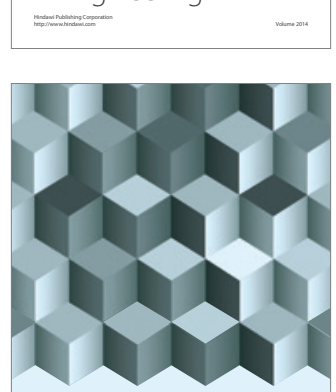

Journal of

Function Spaces
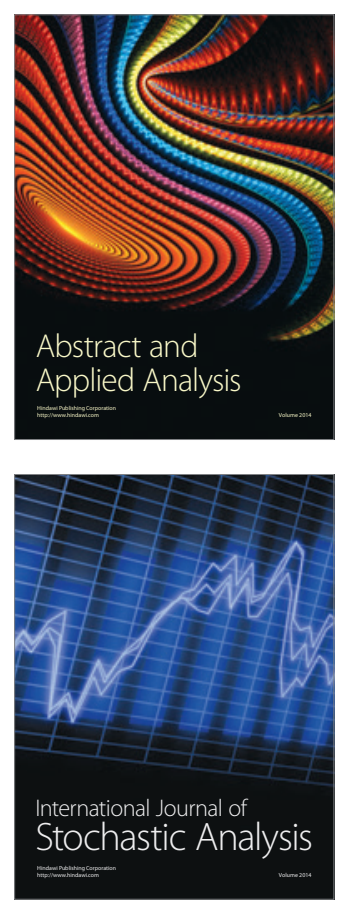

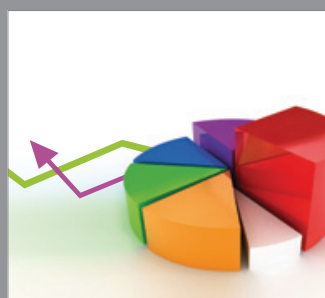

ournal of

Probability and Statistics

Promensencen
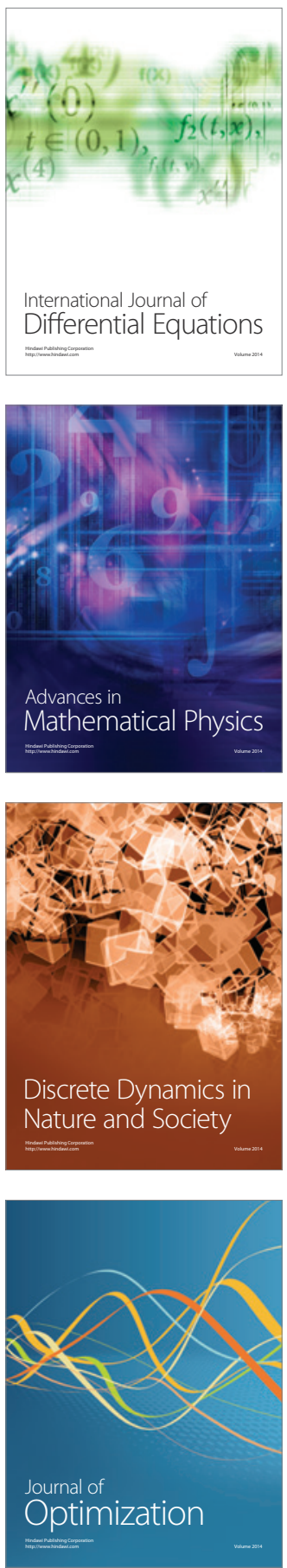